\title{
StoryMINE: A System for Multiplayer Interactive Narrative Experiences
}

\author{
Callum Spawforth, Nicholas Gibbins, and David E. Millard \\ University of Southampton, Southampton, United Kingdom, \\ (cs14g13, nmg, dem) @soton.ac.uk
}

\begin{abstract}
Multiplayer Interactive Narrative Experiences (MINEs) are interactive authored narratives in which multiple players experience distinct narratives (multiplayer differentiability) and their actions influence the storylines of both themselves and others (inter-player agency). Little research has been done to explore the possibilities of this type of narrative, and no complete model nor system exists. In this paper we introduce a model for MINEs based on sculptural hypertext and describe its implementation in a prototype system: StoryMINE. Then using a number of working narrative scenarios we demonstrate that the model and system supports a variety of inter-player interactions. It is our hope that this system provides a platform for the creation and reading of MINEs, and that this in turn creates opportunities for further research into this novel form of digital storytelling.
\end{abstract}

Keywords: Sculptural Hypertext · Narrative Systems · Multi-Player

\section{Introduction}

Interactive Narrative (IN) research has traditionally focused on singleplayer experiences, with little done to explore the potential of multiplayer storytelling [14]. Support for narratives where every player experiences a distinct narrative (multiplayer differentiability [14]) and each player has the ability to influence the narratives of other players (inter-player agency) is especially rare. We define the term Multiplayer Interactive Narrative Experiences (MINEs) as interactive authored narratives that demonstrate both multiplayer differentiability and inter-reader agency.

MINEs make new types of story possible. For example, consider a detective story in which the players experience the different narratives of a detective and a criminal. The clues available to the the detective might be defined by the actions of the criminal, while the ultimate fate of the criminal might be decided by the effectiveness of the detective's investigation. Alternatively, consider the story of a player exploring a haunted house, where the player ultimately meets their demise. The nature of the next player's haunting could depend upon the decisions the previous player made when exploring the house. Here, the players take on the role of the same character, but with a different story due to the actions of players in previous tellings. 
Unfortunately little research exists that explores these ideas, frequently adopting a collaborative authoring or group-based perspective [4]. The result is a lack of models or systems with which to explore this space. While some attempts have been made to address this, they either lack sufficient detail to be implemented (for example, Riedl et al.'s MuSE [14]), or have implementations that are unclear and are now unobtainable (for example, Fairclough and Cunningham's case based story engine [3]). This is a clear barrier to further research.

In this paper, we present a narrative model based on sculptural hypertext[1] that enables the design of MINEs and a prototype system supporting this model, StoryMINE, to our knowledge the first of its type. We then present a scenariobased evaluation that demonstrates StoryMINE's support for all of the interaction types previously identified in non-narrative multi-player games [17].

\section{Background}

Few multiplayer IN systems exist, none of which completely support MINEs. The most relevant aspects of these systems are the extent to which the narrative is constrained by the author (known as authorial intent [15]), their support of multiplayer differentiability, and their level of inter-player agency.

Peinado and Gervas [4] adopt a generative approach, using case-based reasoning (CBR) to plan a sequences of scenes using Propp's Morphology of the Folk Tale [13]. However, the system treats the group of players as a single entity, resulting in a lack of multiplayer differentiability. Fairclough and Cunningham [3] also adopt a CBR based approach, using an experience manager [15] to generate an individual story for each player. However, the system treats inter-player agency as an emergent property. The efficacy of their system for multiplayer was never tested, and the system itself is now unavailable.

Manninen et al. [10] present an emergent [9] approach to multiplayer storytelling where the story arises from social conflict between players. The system assigns a role, task, objective, threat and item of knowledge to each player. Each of which brings them into conflict with other players. However, once these traits are assigned the system takes a hands-off approach, allowing the narrative to emerge from the conflict between players trying to achieve their goals.

Social Shark [1] represents one of few authored approaches to multiplayer interactive narrative. A collaborative system based on sculptural hypertext [1, $12,7]$, it represents fragments of the narrative as a deck of cards. Each card can be played when a set of card-specific constraints are satisfied. Each player has a hand of cards, taking it in turns to play cards and build the narrative. Unfortunately, this system does not offer multiplayer differentiability as the players share a common narrative. However, it is one of few highly authored examples.

The Multiplayer Storytelling Engine (MuSE) [14] also adopts an authored approach and closest to supporting what we define as MINEs. Based on Coloured Petri Nets [8], it encodes scenes as places and players as tokens, while transitions encode the intended progression of players through the narrative. Notably, the system uses a partial-order planner to handle player actions which exceed 
the authored bounds of the story. Crucially, the model is designed to handle multiplayer differentiability, however it doesn't explicitly consider inter-player agency, instead supposing a shared virtual environment where players interact. Thus it only provides a broad description of the narrative (used for the planner), rather than a complete design.

In our own ICIDS 2017 paper, we previously considered the topic of multiplayer storytelling [17] and argued that it should be possible to translate the interaction characteristics of multi-player games to apply to multi-player narratives, and that these could be supported by a sculptural hypertext engine. It is this principle that underlies our work, and forms the basis of our evaluation.

\section{Multiplayer Model and StoryMINE System}

Our model for MINEs is based on sculptural hypertext. This is an approach to narrative based around rules and constraints, and has also been described as quality-based structure [16]. Traditional hypertext, described as calligraphic by Bernstein [1], consists of a graph of nodes connected by links explicitly added by an author. Sculptural hypertext $[1,12,7]$ inverts this by making every node potentially accessible from every other node. Nodes are assigned a set of constraints that must be satisfied within the current story, in order for the node to be visitable. For example, a "Free the prisoner" node might only be available when the constraint "Dragon has been dealt with" is satisfied. To satisfy these conditions, each node is capable of making assertions about the story world when it is visited. This collection of assertions is known as the story state. In this case, visiting the nodes "Slay the Dragon" or "Evade the Dragon" would satisfy "Dragon has been dealt with", and more complex structures are possible.

By default, sculptural hypertext assumes a single reader with their own instance of the story. But our multiplayer model extends this to support MINEs, adding mechanisms for inter-player agency and multiplayer differentiability. Interplayer agency is achieved by making multiple players share the same story instance and reading state. This enables the actions of any player to alter the shared state, changing the nodes available to other players.

However, in our earlier analysis of interaction in multiplayer games, the game Dark Souls demonstrated the interesting case where players exist within their own game world, but could trigger interactions that manifested in the game worlds of others. For example, a player might place a symbol on the ground that appears in the other worlds ${ }^{1}$. The game Moirai ${ }^{2}$ also demonstrates this, with content generated by previous players appearing in the games of subsequent players. In sculptural hypertext, this cannot be accomplished by simply sharing a single story-world, as any changes will be localised to that world.

To resolve this, a new type of state is introduced that can be accessed from any instance of a given story. By modifying this state, other instances of the

\footnotetext{
${ }^{1}$ http://darksouls.wikidot.com/orange-guidance-soapstone

${ }^{2}$ https://kotaku.com/moirai-is-an-adventure-game-with-a-killer-twist-1795897859
} 
story can be influenced, allowing inter-player agency between any of the readers of a story - even if only a single role exists within the story.

The challenge with multiplayer differentiability in authored narrative is presenting different perspectives on the narrative to different players. In systems using virtual environments, this is achieved with locations $[3,10]$ or scenes $[4,14]$. A similar form of narrative separation can't be used here as all state is shared, providing no strict division of the narrative space. To solve this, we introduce roles, unique identifiers defined by the author that are intended to represent a specific point of view. A role is assigned to each player, and an author can specify which parts of the story each role should experience using constraints.

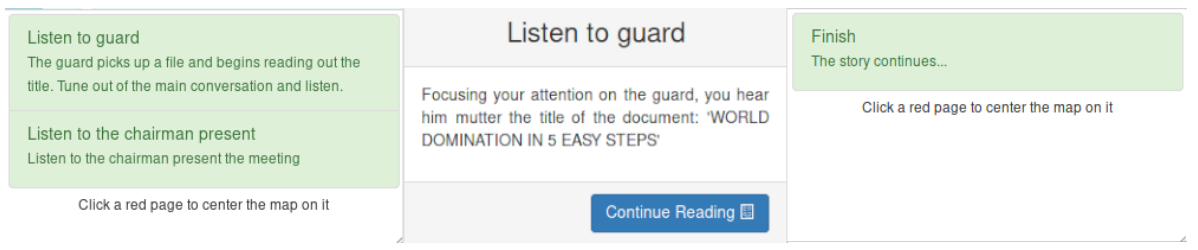

Fig. 1. StoryMINE: Making a choice, reveals the text, and changes the available choices

The StoryMINE system (shown in Fig. 1 running one of the exemplars) is one possible implementation of this model. Rather than creating a sculptural hypertext engine from scratch, it extends the open-source StoryPlaces platform to support MINEs. StoryPlaces is a web-based platform for location-based narratives based on sculptural hypertext, although the use of location is not a requirement. A full description can be found in [6].

StoryMINE implements the model but it embeds the role selection mechanism into the story itself, providing nodes with the ability to set the a player's role upon reading. This allows the author the freedom to incorporate role selection into the narrative itself, and enables role changes as the story progresses.

Three key properties of sculptural hypertext were identified that are are implicit for a single player hypertext, but needed to be explicitly supported for multi-player StoryMINE. These are: atomicity, consistency and isolation, analogous the ACID properties of database transactions [5].

Atomicity states that visiting a node either succeeds or fails completely; it should be impossible for a reader to view the content of a node if all the actions for that node haven't successfully executed. Consistency states that synchronising state should always leave the hypertext in a valid state. In practice, this means that the system should never allow a node to be visited whose constraints are not satisfied. Isolation states that seemingly simultaneous node visits by multiple players should behave as if performed sequentially.

The the full StoryMINE system is available as Open Source on GitHub ${ }^{3}$.

\footnotetext{
$\overline{3}$ https://github.com/StoryMINE
} 


\section{Evaluation}

We have performed a scenario-based evaluation of StoryMINE, considering each of the possible interaction characteristics from multi-player games [17]. We designed three brief exemplar narratives each featuring a subset of those characteristics and created them in StoryMINE, together they demonstrate the coverage of those characteristics by the system. These characteristics were:

Likelihood, the chance of an interaction occurring as the result of a given player action. Interaction type, describes whether an interaction merely provides additional information to the other player, or whether it has a mechanical effect. Synchronicity, whether an interaction requires the virtual presence of another player, typically because it is part of an action/response pair or sequence. Awareness, the extent to which a player knows they've been affected by another player's action. Feedback, the extent to which a player knows their action has affected another player. And Identifiability, whether a player knows the source or target of an interaction.

Table 1. Overview of the interaction characteristics demonstrated by the exemplars

\begin{tabular}{c|c|c|c} 
& Exemplar 1 & Exemplar 2 & Exemplar 3 \\
\hline Likelihood & Guaranteed & Guaranteed & Possible \\
\hline Type & Mechanical & Mechanical & Informational \\
\hline Synchronicity & Sync. & Sync. & Async. \\
\hline Awareness & Always & Always & Possible \\
\hline Feedback & Always & Never & Possible \\
\hline Initiator Identifiable & Always & Possible & Always \\
\hline Recipient Identifiable & Always & Never & Possible
\end{tabular}

\subsection{Exemplar Narrative Scenarios}

The three exemplar scenarios are presented below. In each case the narrative scenario is described and a figure shows the implementation in StoryMINE. The figure consists of nodes, locking relationships and unlocking relationships. An unlocking relationship specifies that the destination node requires that the source node has been visited before it can be seen, while a locking relationship is where the destination node is unavailable if the source node has been visited.

Table 1 shows the mapping of characteristics to the three exemplars. 


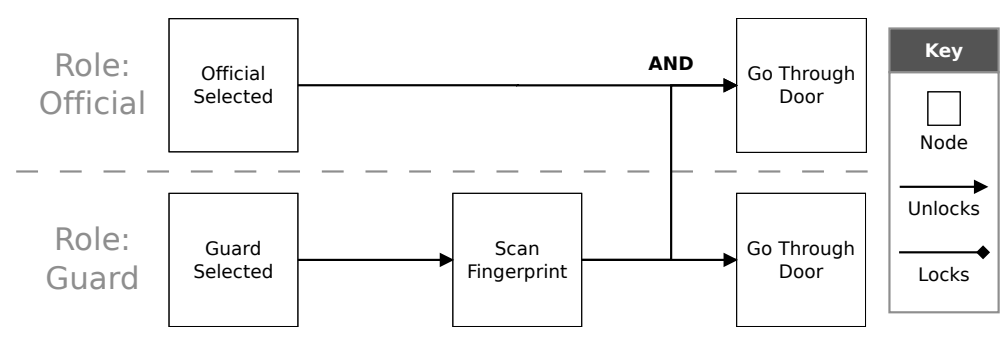

Fig. 2. Structure of Exemplar 1

Exemplar 1: The Secure Room A high-ranking Official is being escorted through a government facility by a Guard. Their destination is a locked metal door marked 'TOP SECRET'. In order to enter the room, the Guard must scan their fingerprint to unlock the door.

Exemplar 1 demonstrates a guaranteed interaction, triggered by the guard scanning their fingerprint. As the Official must visit a node that is unlocked by the Guard, the Official will always experience the interaction.

This gating technique [7] can also be used to synchronise players that may be reading at different rates. Here, it forces a slow Guard to catch up to a quick-reading Official (which could be important if the following scene was synchronous). However, we imagine that overuse of this technique could lead to a poor experience if the narrative of one player is frequently blocked by the other.

Whether the players realise an interaction has occurred depends on the author. If the text makes it clear that another player was involved (such as through an extra-diegetic [11] message) it would be explicit awareness/feedback, if not then deductive. In a story with more roles the author could also use the text for identifiability, naming the Guard/Official, or could leave it ambiguous. In this case, as written, both players are always aware of the interaction and always identifiable

Exemplar 2: The Meeting The Official and the Guard enter the 'TOP SECRET' room to find the other meeting attendees waiting around a large table that takes up the center of the room. As the Official goes to sit down, they knock some precariously perched files off of the table edge. The Guard has a short time to catch the files in order to avoid picking them up from the floor.

Exemplar 2 demonstrates the second way that a narrative might contain synchronous interactions, using time sensitive nodes. When the Official knocks the files from the desk, a new node is opened up to the Guard for 5 seconds, allowing them to catch the files mid-air. With this mechanism, the time-limited node may expire before the player is able to view it due to them being too far behind in the narrative. This could be mitigated by frequent use of synchronisation mechanisms, such as the gating used in Exemplar 1.

The Guard will always be aware that the Official has knocked the files off of the table due to the content of the nodes. In contrast, the Official receives 


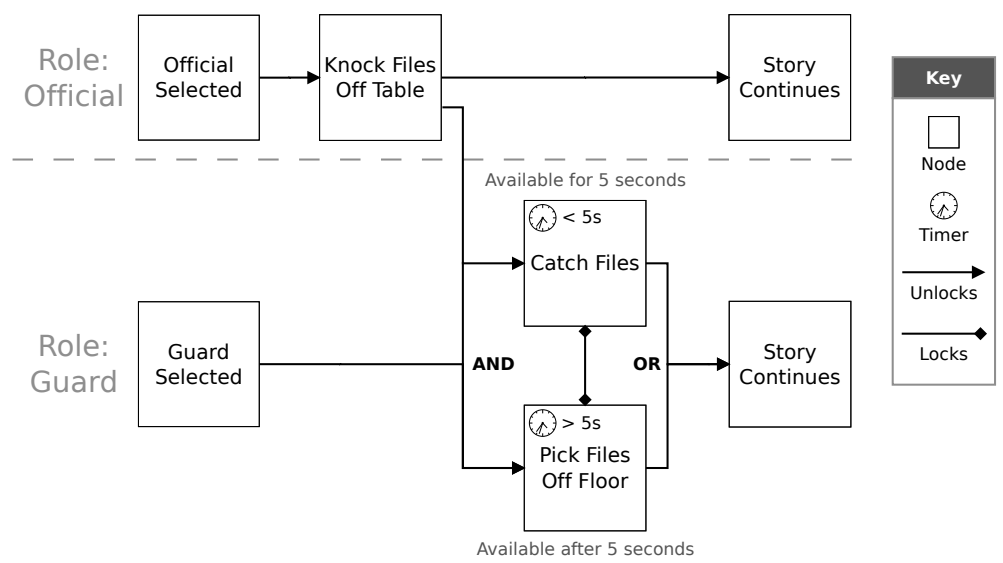

Fig. 3. Structure of Exemplar 2

feedback only if the story later refers to the guard catching or collecting the files. In the short exemplar shown this is omitted, so there is never any feedback. As a result, the guard is never identifiable.

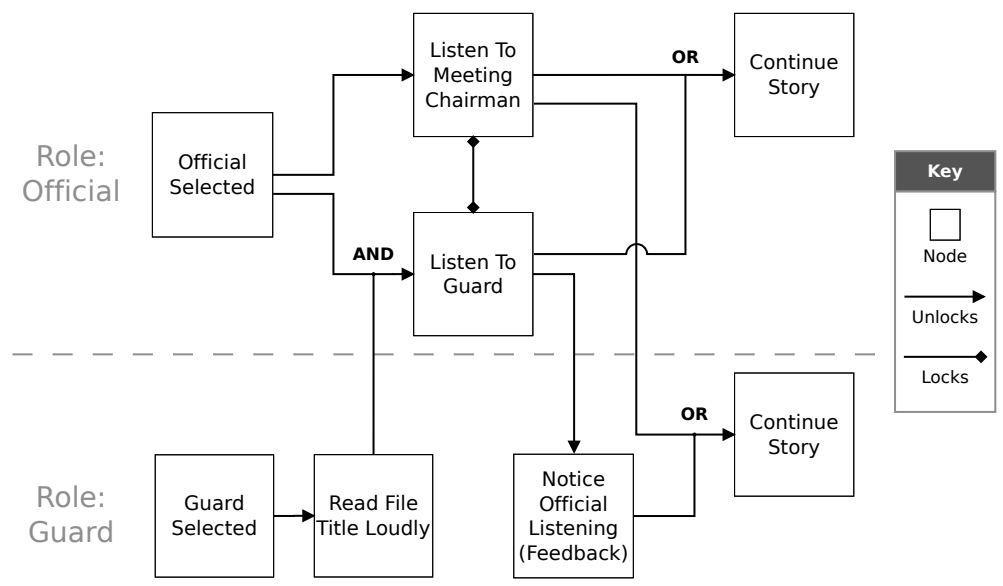

Fig. 4. Structure of Exemplar 3

Exemplar 3: The Important File Files in hand, the Guard places them back down on the table, reading out the title of the top file as they do so. The Official either listens to the chairman of the meeting talk, or listens to the guard, gaining the Guard's attention if they do so. Having placed the files back on the table, the Guard goes and stands by the door. 
In Exemplar 3 the interaction triggered by the Guard reading the file causes the "Listen to Guard" node to appear for the Official. If the Official chooses to listen to the Guard, a new node is opened up for the Guard informing them of their impact on the Official's narrative. This provides explicit feedback. However, if the Official is presented with the choice and chooses not to listen to the Guard an interaction has still occurred, but the Guard won't receive any feedback.

This is also an informational interaction. For the Official, the narrative past this point isn't structurally impacted, however they may have been provided with information that could influence their decisions later.

\section{Discussion and Conclusions}

In this paper, we introduced the term MINEs as multiplayer authored interactive narratives that possess multi-player differentiability and inter-player agency. We briefly explored existing approaches to multiplayer interactive narrative before concluding that none offer full support for MINEs. Following this, we outlined a model and prototype system for MINEs based on sculptural hypertext and then demonstrated that the system supports the full variety of interaction types.

This system represents, to our knowledge, the first usable platform for MINEs, providing a basis for future work in multiplayer storytelling. By using a sculptural hypertext model, the system benefits from existing research into authoring [2] and a known ability to support a variety of interactive narratives.

Our model for MINEs makes no assumptions about how the narrative will be presented to the readers. Existing work $[4,3,10,14]$ assumes a particular gameworld through which narrative elements and interactions are presented. StoryMINE's requires no gameworld at all, and as the exemplars show can support players through an entirely text-based narrative experience.

However, as a consequence of needing to explicitly model individual interactions, there is likely to be an increase in authorial difficulty. Since each additional role requires interactions with each other role, there may be a non-linear increase in both quantity of content and structural complexity. This suggests a distinct challenge in scaling this system to a larger number of players.

Adding to the authoring challenges are concurrency problems such as starvation and deadlock - these are fully managed at the system layer, but can still appear in the narrative structure. For example, if gating is not employed, with one player able to finish before another, then the second player will be starved of agency over the first. However, if gating is employed there is the possibility of a deadlock, where the narrative cannot progress due to a lack of available nodes. Further work in MINE authoring will need to consider these challenges.

The provision of a model and a usable, open-source system enables the creation of publicly readable MINEs for the first time. This allows for research into the possibilities for multi-participant stories, particularly in terms of structure, poetics and authoring. For our future work we intend to develop and evaluate an extended MINE story, and it is our hope that the model, system, and examples will encourage further research into this novel area of digital storytelling. 


\section{References}

1. Bernstein, M.: Card Shark and Thespis: Exotic Tools for Hypertext Narrative. In: Proceedings of the 12th ACM Conference on Hypertext and Hypermedia. pp. 41-50. HYPERTEXT '01, ACM, New York, NY, USA (2001). https://doi.org/10.1145/504216.504233, http://doi.acm.org/10.1145/504216.504233

2. Bernstein, M.: Storyspace 3. In: Proceedings of the 27th ACM Conference on Hypertext and Social Media. pp. 201-206. HT '16, ACM, New York, NY, USA (2016). https://doi.org/10.1145/2914586.2914624, http://doi.acm.org/10.1145/2914586.2914624

3. Fairclough, C., Cunningham, P.: A multiplayer case based story engine. Tech. rep., Trinity College Dublin, Department of Computer Science (2003), http://www.cs.tcd.ie/publications/tech-reports/reports.03/TCD-CS-2003-43.pdf

4. Gerv?s, P., Peinado, F.: Transferring game mastering laws to interactive digital storytelling. In: International Conference on Technologies for Interactive Digital Storytelling and Entertainment. pp. 48-54. Springer (2004), http://link.springer.com/chapter/10.1007/978-3-540-27797-2_7

5. Haerder, T., Reuter, A.: Principles of Transaction-oriented Database Recovery. ACM Comput. Surv. 15(4), 287-317 (Dec 1983). https://doi.org/10.1145/289.291, http://doi.acm.org/10.1145/289.291

6. Hargood, C., Weal, M., Millard, D.: The storyplaces platform: Building a webbased locative hypertext system. In: Proceedings of ACM Hypertext 2018. ACM (Apr 2018), https://eprints.soton.ac.uk/421122/

7. Hargood, C., Weal, M.J., Millard, D.E.: Patterns of Sculptural Hypertext in Location Based Narratives Categories and Subject Descriptors. Proceedings of the 27th ACM Conference on Hypertext and Social Media pp. 61-70 (2016)

8. Jensen, K.: Coloured Petri Nets: Basic Concepts, Analysis Methods and Practical Use. Volume 1. Monographs in Theoretical Computer Science. An EATCS Series, Springer-Verlag, Berlin Heidelberg, 2 edn. (1996), //www.springer.com/gb/book/9783540609438

9. Louchart, S., Aylett, R.: Narrative theory and emergent interactive narrative. International Journal of Continuing Engineering Education and Life Long Learning 14(6), 506-518 (Jan 2004). https://doi.org/10.1504/IJCEELL.2004.006017, http://www.inderscienceonline.com/doi/abs/10.1504/IJCEELL.2004.006017

10. Manninen, T., Vallius, L., Kujanp??, T.: Plot Clusters ? Intertwined and Re-playable Storyline Components in a Multiplayer RPG. In: Technologies for Interactive Digital Storytelling and Entertainment. pp. 265-276. Lecture Notes in Computer Science, Springer, Berlin, Heidelberg (Dec 2006). https://doi.org/10.1007/11944577_27, https://link.springer.com/chapter/10.1007/11944577_27

11. Mason, S.: On Games and Links: Extending the Vocabulary of Agency and Immersion in Interactive Narratives. In: Interactive Storytelling. pp. 25-34. Lecture Notes in Computer Science, Springer, Cham (Nov 2013). https://doi.org/10.1007/978-3319-02756-2_3, https://link.springer.com/chapter/10.1007/978-3-319-02756-2_3

12. Millard, D.E., Hargood, C., Jewell, M.O., Weal, M.J.: Canyons, deltas and plains. Proceedings of the 24th ACM Conference on Hypertext and Social Media - HT '13 pp. 109-118 (2013). https://doi.org/10.1145/2481492.2481504, http://dl.acm.org/citation.cfm?id=2481492.2481504

13. Propp, V.I.: Morphology of the Folktale, vol. 9. University of Texas Press (1968) 
14. Riedl, M., Li, B., Ai, H., Ram, A.: Robust and Authorable Multiplayer Storytelling Experiences. In: AIIDE (2011)

15. Riedl, M.O., Bulitko, V.: Interactive Narrative: An Intelligent Systems Approach. AI Magazine 34(1), 67 (Dec 2012), https://www.aaai.org/ojs/index.php/aimagazine/article/view/2449

16. Short, E.: Beyond Branching: Quality-Based, Salience-Based, and Waypoint Narrative Structures (Apr 2016), https://emshort.blog/2016/04/12/beyond-branchingquality-based-and-salience-based-narrative-structures/

17. Spawforth, C., Millard, D.E.: A framework for multi-participant narratives based on multiplayer game interactions. In: Interactive Storytelling: 10th International Conference, ICIDS 2017, Madeira, Portugal, November 14-17, 2017. Proceedings (Sep 2017) 